\title{
LA COOPERACIÓN INTERNACIONAL EN CIENCIA Y TECNOLOGÍA DE CHINA. \\ EL CASO DE LAS VACUNAS CONTRA EL COVID-19 EN LA RELACIÓN CON ARGENTINA
}

\section{Carla Oliva*}

\section{Resumen}

El actual contexto internacional, caracterizado por el reordenamiento geopolítico, está atravesado por la pandemia del COVID-19.

En el marco de la disputa hegemónica entre China y Estados Unidos, la ciencia y tecnología (CyT) desempeña un papel central, en tanto la política de China encaminada a transformar al país en la vanguardia de la tecnología mundial para 2049 y la consecuente reducción de la brecha tecnológica con Estados Unidos están en el eje de la competencia entre ambas potencias.

Este artículo analiza la cooperación en materia de vacunas desplegada por China como cooperación internacional en CyT, explicando su articulación con el planeamiento estratégico de China, y los alcances de esta cooperación con Argentina.

Palabras clave: Ciencia y Tecnología - Cooperación Internacional - vacunas -

\section{MEDICINES PATENTS \& COVID-19: REFLECTIONS ON ACCESS TO HEALTH IN MULTILATERAL REGULATIONS AND INTERNATIONAL COOPERATION IN THE FRAMEWORK OF THE PANDEMIC}

\begin{abstract}
The current international context, characterized by geopolitical reordering, is crossed by the COVID-19 pandemic.

In the framework of the hegemonic dispute between China and the United States, science and technology (S\&T) plays a central role, as China's policy aimed at transforming the country into the forefront of world technology by 2049 and the consequent reduction in the technological gap with the United States is at the center of competition between the two powers.

\footnotetext{
* Docente de Política Internacional de la Facultad de Ciencia Política y Relaciones Internacionales de la UNR. Coordinadora del Grupo de Estudios sobre China en dicha Unidad Académica. Magister en Integración y Cooperación Internacional (CERIR-CEI-UNR). Correo electrónico: carlaoliva@yahoo.com. ORCID: https://orcid.org/0000-0002-5358-6602
}

Esta obra se encuentra bajo la Licencia Creative Commons Reconocimiento - Compartir Igual (by-sa) 
This article analyzes the cooperation in the field of vaccines deployed by China as international cooperation in S\&T, explaining its articulation with China's strategic planning, and the scope of this cooperation with Argentina. vaccines

Key words: science and technology - International Cooperation -

TRABAJO RECIBIDO: 10/05/2021 -TRABAJO ACEPTADO: 05/06/2021 


\section{Consideraciones iniciales}

La cooperación internacional en Ciencia y Tecnología (CyT) ha ocupado un lugar relativamente marginal en la disciplina de las Relaciones Internacionales. La mayor parte de las investigaciones sobre cooperación abordan cuestiones vinculadas con el desarrollo económico y social que tratan indirectamente la cooperación técnica o científica.

Entre los especialistas en cooperación internacional en CyT, Kern (2009) evalúa su rol como elemento de inserción en el escenario internacional y explica que la política internacional en CyT se conforma a partir de la intersección de cuestiones de política exterior y de política científica.

El actual escenario internacional nos invita a profundizar los análisis sobre la materia, particularmente porque la pugna hegemónica entre China y Estados Unidos se encuentra atravesada por la dimensión científico-tecnológica. Sumado a ello, la situación de crisis sanitaria generada por la pandemia del COVID-19 ha conducido a la necesidad de vacunas para enfrentar la enfermedad. Una vez desarrolladas las mismas, la cuestión de su accesibilidad se volvió vital. Su distribución a gran escala se transformó en un instrumento diplomático con efectos geopolíticos cuyo impacto está transcurriendo en nuestros días. En relación a esta cuestión, la cooperación internacional en CyT desempeña un papel clave, por cuanto el desarrollo de vacunas se ubica en el marco de los procesos de CyT.

El contexto global ha puesto de relieve que la cooperación en CyT en relaciones internacionales no sólo es importante por sus aplicaciones en el área militar, de la energía, la astronomía o la biotecnología. Las vacunas suponen una nueva área de cooperación en CyT cuyas modalidades, objetivos y significación deben ser materia de estudio. Por lo tanto, a las áreas mencionadas anteriormente debemos sumar la sanitaria, específicamente las vacunas.

A raíz de la importancia que adquirieron los antígenos en el contexto de pandemia, comenzamos a escuchar con más frecuencia el término "diplomacia de vacunas" para hacer referencia al rol central de la distribución y accesibilidad de las vacunas contra el COVID-19. En este sentido, es importante realizar una aclaración conceptual, ya que en las relaciones internacionales encontramos especialistas que se han referido a la relevancia de la CyT en la diplomacia. Vera y Colombo (2020) analizan la convergencia entre cooperación en CyT y política exterior y presentan el concepto "diplomacia científica", de uso reciente en la academia y las agencias gubernamentales. De acuerdo con las autoras, la finalidad de la diplomacia científica es "promover los objetivos de desarrollo nacional y política exterior de un Estado y contribuir con el incremento de su poder y prestigio en el sistema internacional a través de la generación de poder blando o soft power, promoviendo sus capacidades científico-tecnológicas para resolver los problemas que enfrenta la humanidad" (Vera y Colombo, 2020, p. 18).

En el marco de la diplomacia científica así entendida, este artículo analiza la cooperación en materia de vacunas desplegada por China como cooperación internacional 
en CyT, explicando su articulación con el planeamiento estratégico de China y los alcances de esta cooperación con Argentina.

\section{La articulación entre innovación, cooperación internacional en CyT y vacunas}

Los descubrimientos y avances vinculados a la CyT han sido un componente central en la identidad china desde hace mucho tiempo. En la larga etapa imperial, China se auto percibía y era vista por sus vecinos como el "centro radiante" de una civilización desde la que fluían conocimientos científicos y técnicos que le permitían influir en su región. Además, sus aportes históricos en términos de avances científicos, como la imprenta, el papel y la brújula, han sido muy significativos y de alcance global. Tal es la relevancia asignada a la CyT que, para los académicos y gobernantes chinos, el atraso tecnológico atribuido a la última dinastía fue una de las causas de la situación que condujo a la "etapa de la humillación colonial", situada temporalmente en los cien años anteriores a la revolución comunista de 1949.

A partir de la proclamación de la República Popular, los planes quinquenales han orientado las políticas gubernamentales y, progresivamente, han incluido y visibilizado el rol que el gobierno asigna a la CyT. De esta manera, el Plan Quinquenal 2021-2025 proyecta aumentar el gasto en CyT, crear mayores vínculos entre la academia y la industria y potenciar la ciencia básica. Los recursos institucionales, materiales y humanos destinados a alcanzar esos objetivos se incrementan constantemente. Vale decir que el aumento de las capacidades evidenciadas por China tiene un peso considerable en su posicionamiento como potencia y constituye una parte importante de las preocupaciones de Estados Unidos en su relación con ese país.

En el marco de su planeamiento estratégico, el gobierno ha dado a conocer el plan Made in China 2025 (MIC 2025), en el cual explicita el objetivo de transformarse en la vanguardia de la innovación tecnológica mundial para el año 2049. La propuesta busca una gradual sustitución de la tecnología extranjera en los procesos productivos nacionales y la extensión de la presencia china en esta área a escala global (Balderrama Santander y Trejo Romero, 2018).

Para cumplimentar sus objetivos, el gobierno ha definido una serie de acciones destinadas a fomentar la innovación y generar avances tecnológicos en 10 áreas industriales puntuales en las cuales pretende convertirse en líder mundial. Ellas son: la nueva generación de inteligencia artificial (IT), máquinas y robots computarizados de alta gama, equipo de aviación y espacial, equipo de ingeniería marítima y barcos de alta tecnología, equipo avanzado de transporte ferroviario, vehículos de ahorro de energía y de nueva energía, equipo de energía, equipo agrícola, nuevos materiales y biomedicina y equipo médico de alto rendimiento.

De las áreas industriales antes mencionadas nos interesa explicar la relación entre la biomedicina y las vacunas. De acuerdo con la Real Academia Española (RAE), la biomedicina es el conjunto de disciplinas como la bioquímica, la biología molecular y celular y la genética que desempeñan un papel fundamental en la medicina actual. Mientras 
que las vacunas en general son el mayor logro de la biomedicina, aquellas destinadas a la inmunización contra el COVID-19, en particular, dan testimonio del nivel de evolución de las investigaciones en esta materia. Nunca antes en la historia de la humanidad se habían desarrollado vacunas contra una enfermedad con tanta celeridad. Obviamente, esto fue posibilitado por los avances en CyT.

Durante el año 2020, laboratorios de Estados Unidos, el Reino Unido, Rusia, India y China desarrollaron vacunas. Hasta el momento China ha desarrollado tres vacunas para combatir al COVID-19. La primera, de la farmacéutica estatal Sinopharm y el Instituto de Productos Biológicos de Pekín, es una vacuna "inactivada" - elaborada de una proteína $\mathrm{u}$ otros pequeños fragmentos tomados de un virus o bacteria que no produce enfermedad, pero genera anticuerpos-. La segunda, del laboratorio Sinovac Biotech, tiene las mismas características que la de Sinopharm. La tercera, del Instituto de Biotecnología de Pekín y la farmacéutica CanSino Biologics Inc, usa la tecnología del adenovirus y, a diferencia de las anteriores, requiere una sola dosis.

Pekín ha comenzado a distribuir inoculantes a gran escala. Según algunos cálculos, sus laboratorios vendieron o recibieron pedidos internacionales por más de 800 millones de dosis. Además, prometió 10 millones adicionales para el Fondo Global de Acceso para Vacunas COVID-19 conocido como Covax, herramienta para distribuir vacunas independientemente de los recursos económicos de cada país, promovido por la Organización Mundial de la Salud, la Comisión Europea y el gobierno de Francia (Oliva, 2021).

La inclusión de la biomedicina en el MIC 2025 da cuenta de la estrategia china en la materia. Como tal, fija una meta y orienta la distribución de recursos humanos y materiales. Pero, además, el desarrollo de la vacuna contra el COVID-19 y su reparto a diferentes países es una manifestación de la expansión de la CyT china a diferentes regiones del mundo.

Siguiendo a Vera y Colombo (2020), la vacuna ha sido un instrumento de la diplomacia científica de China en la medida en que representa un caso de convergencia entre la política exterior y la cooperación en CyT. Se puede afirmar que le permite a China aumentar su poder y prestigio internacional a partir de la generación de poder blando, en tanto sus capacidades científico-tecnológicas están disponibles para resolver los problemas de la humanidad.

\section{La cooperación en CyT en materia de vacunas entre China y Argentina}

La cooperación en CyT entre China y Argentina data del establecimiento de relaciones bilaterales en 1972, con intercambio de expertos de ambos países. Las relaciones en esta dimensión fueron cobrando densidad en función de la firma de diferentes acuerdos. En este contexto, en 1980 se firmó el primer Convenio de Cooperación Científica y Técnica que funcionó como marco general de las relaciones en dicha dimensión y al que se agregaron sucesivos tratados sobre la materia en áreas específicas. En 2014, en el marco 
de la suscripción de la Asociación Estratégica integral entre los dos países, se firmó un Acuerdo Estratégico de Cooperación Tecnológica.

A fines de 2019, el Consejo Nacional de Investigaciones Científicas y Técnicas (CONICET) de Argentina y la Academia China de Ciencias Sociales acordaron la creación de un Centro Conjunto de Ciencia y Tecnología para favorecer la colaboración y comunicación entre las instituciones y las comunidades científicas de ambos países (CONICET, 2019). Las áreas prioritarias de dicho Centro son Astronomía, Biomedicina, Ciencias Ambientales, Ciencias de la Tierra y la Atmósfera y Estudios Polares. De acuerdo con la Oficina de Cooperación Internacional del CONICET (Comunicación personal, 3 de junio de 2021), este Centro es una iniciativa que aún está en proceso.

Siendo la biomedicina un área prioritaria del mencionado Centro, y en la cual se enmarca el desarrollo de vacunas, su incorporación puede ser enlazada con los objetivos del MIC 2025. Podemos decir que estamos ante una oportunidad de avanzar en la cooperación en un área estratégica.

Entre los especialistas en la materia, Malacalza (2019) analiza las modalidades y casos de cooperación en ciencia, tecnología e innovación (CTI) sino-argentina y la sitúa en el nivel de las tecnologías de punta, incluyendo astronomía, biotecnología y nanotecnología, tecnología aeroespacial, energías renovables, tecnología nuclear y tecnologías de la información (TIC). El autor realiza su análisis en función de cuatro modelos de cooperación surgidos a partir del cruzamiento de dos variables: la participación empresarial y la transferencia tecnológica. Los modelos son la explotación y uso científico, el proyecto científico conjunto, la venta llave en mano y el triángulo de Sábato1.

Por su parte, la también académica argentina Haro Sly (2019) considera que las áreas de cooperación en CyT más relevantes entre China y Argentina son biotecnología, alimentos y sector nuclear. En función de su análisis, elabora propuestas para nuestro país, entre las que se destaca la sugerencia de enfatizar temas estratégicos que incluyan tanto la transferencia de tecnología como el aprendizaje de gestión para el escalamiento de las investigaciones científicas. En ese marco sobresale la importancia de los parques industriales de tecnología de punta.

En este artículo situamos a la más reciente cooperación en vacunas como cooperación en CyT. Entendemos que esta dimensión incluye los testeos realizados en Argentina, la compra de vacunas y los anuncios sobre la producción de vacunas chinas en nuestro país.

En agosto de 2020 el Ministerio de Salud de Argentina acordó con el gobierno chino la realización de testeos de la vacuna desarrollada por Sinopharm a un grupo de al menos 500 voluntarios. En septiembre de 2020 la Fundación Huésped y la Fundación Vacunar dieron inicio a la fase 3 de testeos de la vacuna china inactivada (Ámbito Financiero, 2020).

\footnotetext{
${ }^{1}$ El triángulo de Sábato se refiere a las interacciones entre políticas públicas, actividades productivas y capacidades científico-tecnológicas.
}

Esta obra se encuentra bajo la Licencia Creative Commons Reconocimiento - Compartir Igual (by-sa) 
En febrero de 2021 el Ministerio de Salud de Argentina (2021) autorizó con carácter de emergencia la vacuna Sinopharm. Esta autorización permitió el ingreso de dosis provenientes de la farmacéutica china a nuestro país. De acuerdo con la agencia de noticias Télam (2021a), para el 23 de mayo de 2021 Aerolíneas Argentinas llevaba realizados 5 vuelos hacia Beijing, en los que arribaron al país 3.659.200 de vacunas de la recién mencionada farmacéutica. Sumando a lo anterior, el 28 de mayo de 2021 se anunció la firma de un preacuerdo para la compra de otros 6 millones de dosis adicionales que arribaría a Argentina en los siguientes dos meses (Menegazzi, 2021). Según esa información, a mediados de junio llegarían 2 millones de dosis y en julio los restantes 4 millones.

En relación a la producción de vacunas chinas en Argentina, el 3 de mayo de 2021 la Ministra de Salud mantuvo un encuentro virtual con el embajador chino en Argentina, Zou Xiaoli, el embajador argentino en China, Sabino Vaca Narvaja y directivos de Sinopharm dando inicio a las conversaciones sobre la posible participación argentina en la investigación y producción de vacunas contra el coronavirus (Télam, 2021b). Posteriormente, el embajador Zou Xiaoli afirmó que pudieron alcanzarse acuerdos para la producción de vacunas de Sinopharm en Argentina (Embajada China en Argentina, 2021).

\section{Reflexiones finales}

Las vacunas contra el COVID-19 desarrolladas y distribuidas por China representan una nueva dimensión de cooperación internacional en CyT. A la vez, son la evidencia empírica de los éxitos del planeamiento estratégico de China tanto en términos del objetivo de extender su tecnología a escala mundial como en las áreas de desarrollo tecnológico más importantes para el MIC 2025. En ese sentido, hay una confluencia entre la incorporación de la biomedicina como área prioritaria y las vacunas contra el COVID19 desarrolladas por Beijing.

El planeamiento estratégico chino también se visibiliza en las relaciones bilaterales. La inclusión de la biomedicina en el proyecto del Centro Conjunto en Ciencia y Tecnología entre China y Argentina da cuenta de la proyección de ese planeamiento en sus relaciones con otros países. Al mismo tiempo, la cooperación en vacunas entre ambos países es consistente con la política global de Beijing de extender su CyT y apunta a fortalecer su presencia tecnológica en el resto del mundo.

La cooperación internacional en CyT impacta en el escenario internacional actual. En el reordenamiento geopolítico previo al COVID-19 presenciamos la competencia tecnológica, económica, política y militar entre China y Estados Unidos. La emergencia que supone la pandemia es una oportunidad para estrechar y consolidar vínculos a partir de la cooperación internacional en CyT en materia de vacunas con países estratégicamente importantes y necesitados estas preparaciones para inocular a su población, como es el caso de Argentina. Por lo tanto, las vacunas constituyen una nueva dimensión de análisis en el área de la cooperación internacional en CyT en el contexto global.

Para finalizar, es importante recuperar la idea de que la cooperación en vacunas queda comprendida en la llamada "diplomacia científica" que refuerza la influencia 
mundial de China. Es así que esta dimensión de cooperación internacional en CyT, en tanto componente del poder blando de China, fortalece su imagen en el exterior.

\section{Referencias Bibliográficas}

ÁMBITO FINANCIERO (2020). Coronavirus. Afirman que la vacuna de Sinopharm está siendo un éxito. 21 de octubre. Recuperado de https://www.ambito.com/informaciongeneral/coronavirus/afirman-que-la-vacuna-sinopharm-esta-siendo-un-exito-n5141959

BALDERRAMA SANTANDER, R. Y TREJO ROMERO, A. (2018). Hecho en China 2025 y la autosuficiencia en nuevas tecnologías. Revista Comercio Exterior, (15). Recuperado de https://www.revistacomercioexterior.com/articulo.php?id=506\&t=hechoen-china-2025-y-la-autosuficiencia-en-nuevas-tecnologias

CONICET (2019). El CONICET y la Academia China de Ciencias acuerdan la creación de un Centro conjunto de Ciencia y Tecnología. 3 de diciembre. Recuperado de https://www.conicet.gov.ar/el-conicet-y-la-academia-china-de-ciencias-acuerdan-lacreacion-de-un-centro-conjunto-de-ciencia-y-tecnologia/

$\begin{array}{lcccc}\text { EMBAJADA DE LA REPÚBLICA POPULAR CHINA } & \text { EN LA REPÚBLICA } \\ \text { ARGENTINA } & \text { [@ChinaEmbArg] } & (2021) . & \text { Recuperado } & \text { de } \\ \text { https//twitter com/ChinaEmbArg/status/1389717939843215361? }=08 & \end{array}$

HARO SLY, M. J. (2019). La política científica y tecnológica de China y la cooperación sino-argentina. Ciencia, tecnología Y política,2(3), 029. Recuperado de https://doi.org/10.24215/26183188e029

KERN, A. (2009). La cooperación científica y tecnológica como campo de estudio en las relaciones internacionales. En Jornada Las Relaciones Internacionales hoy: una disciplina en constante movimiento, Jornadas de Relaciones Internacionales, FLACSO, Argentina, 1 y 2 de octubre.

MALACALZA, B. (2019). Cooperación China-Argentina en la Cuarta Revolución Industrial: procesos, nudos críticos y horizontes. En Observatorio América Latina-Asia Pacífico América Latina y Asia entre la revolución digital y la globalización cuestionada, Memorias del IV Seminario Académico América Latina-Asia Pacífico, ALADI,CAF Y CEPAL, Uruguay, 11 de noviembre.

MENEGAZZI, E. (2021). Argentina avanza con la compra de 6 millones de vacunas chinas de Sinopharm, que llegarían entre junio y julio. Infobae. 28 de mayo. Recuperado de https://www.infobae.com/politica/2021/05/28/argentina-avanza-con-la-compra-de-6millones-de-las-vacunas-chinas-de-sinopharm-que-llegarian-entre-junio-y-julio/

MINISTERIO DE SALUD DE LA REPÚBLICA ARGENTINA (2021). Resolución 668/2021. Recuperado de 
https://www.argentina.gob.ar/normativa/nacional/resoluci\%C3\%B3n-688-2021$\underline{347264 / \text { texto }}$

OLIVA, C. (2021). China y la "diplomacia de vacunas". En Análisis CIPEI, (8). Recuperado

https://rephip.unr.edu.ar/bitstream/handle/2133/20574/An\%C3\%A1lisis\%20CIPEI\%208 $\% 20-\% 20$ Oliva.pdf? sequence $=3 \&$ is Allowed $=y$

REAL ACADEMIA ESPAÑOLA (s.f). Biomedicina. En Diccionario de la Lengua Española. Recuperado el 23 de mayo de 2021, Recuperado de https://dle.rae.es/biomedicina?m=form

TÉLAM (2021a). El gobierno avanza en el plan de vacunación con la llegada de nuevas dosis. Recuperado de https://www.telam.com.ar/notas/202105/555168-argentinavacunas-coronavirus-campana-sputnik-v-astrazeneca-sinopharm.html

TÉLAM (2021b). Vizzotti inició diálogo con China e Israel para producir vacunas en Argentina. Recuperado de https://www.telam.com.ar/notas/202105/552884-vizzottiinicio-dialogo-con-china-e-israel-para-producir-vacunas-en-argentina.html

VERA, N. y COLOMBO, S. (2020). Reflexiones en torno a la Cooperación Internacional en Ciencia y Tecnología y la Diplomacia Científica: los casos de Estados Unidos y la República Popular China. En López, M. P. (comp.). Perspectivas sobre la Cooperación Internacional en Ciencia y Tecnología y Universidad. Políticas, prácticas y dinámicas a principios del siglo XXI, CEIPIL, UNICEN, 13-38.

Cómo citar

OLIVA, C. (2021) La Cooperación Internacional en Ciencia y Tecnología de China. El caso de las vacunas contra el COVID-19 en la relación con Argentina. Revista Integración y Cooperación Internacional, 33 (jul-dic), 7-15 